2nd March 2000

Submitted to J. Rheol.

\title{
Molecular Weight Dependent Kernels in Generalized Mixing Rules
}

\author{
WOLFGANG Thimm ${ }^{1}$ \\ Christian Friedrich ${ }^{1}$ 用 \\ TOBIAS Roths ${ }^{1}$ \\ JoSEF HONERKAMP ${ }^{1,2}$ \\ ${ }^{1}$ Freiburger Materialforschungszentrum, Stefan-Meier-Straße 21, D-79104 \\ Freiburg im Breisgau, Germany \\ ${ }^{2}$ Universität Freiburg, Fakultät für Physik, Hermann-Herder-Straße 3, D-79104 \\ Freiburg im Breisgau, Germany
}

\section{Synopsis}

In this paper, a model is proposed for the kernel in the generalized mixing rule recently formulated by Anderssen and Mead (1998). In order to derive such a model, it is necessary to take account of the rheological significance of the kernel in terms of the relaxation behaviour of the individual polymers involved. This leads naturally to consider a way how additional physical effects, which depend on the molecular weight distribution, can be included in the mixing rule. The advantage of this approach is that, without changing the generality derived by Anderssen and Mead (1998), the choice of the model proposed here for the kernel guarantees the enhanced physical and rheological significance of their mixing rule.

*Author to whom all correspondence should be addressed. Electronic mail:chf@fmf.unifreiburg.de 


\section{Introduction}

In an earlier article (Thimm et al. (1999)) an analytical relationship was derived between the relaxation time spectrum and the molecular weight distribution (MWD):

$$
w(m)=\frac{1}{\beta} \frac{\alpha^{(1 / \beta)}}{\left(G_{N}^{0}\right)^{1 / \beta}} \tilde{h}(m)\left(\int_{m}^{\infty} \frac{\tilde{h}\left(m^{\prime}\right)}{m^{\prime}} \mathrm{d} m^{\prime}\right)^{(1 / \beta-1)} .
$$

This relation is based on a generalized mixing rule formulated and analyzed by Anderssen and Mead (1998). Among other things it can be used to calculate the MWD $w(m)$ once an estimate of the relaxation time spectrum $h(\tau)$ has been derived. The corresponding inverse relationship is given by:

$$
\frac{\tilde{h}(m)}{G_{N}^{0}}=\frac{\beta}{\alpha} w(m)\left[\int_{m}^{\infty} \mathrm{d} m^{\prime} \frac{w\left(m^{\prime}\right)}{m^{\prime}}\right]^{\beta-1} .
$$

In these equations, $\beta$ is the generalized mixing parameter, $G_{N}^{0}$ the plateau shear modulus and $\alpha$ is the scaling exponent

$$
\tau=k m^{\alpha}, k=\text { const., }
$$

where $\alpha \approx 3.4$, which is normally determined experimentally. Let $\tilde{h}(m) \equiv$ $h(\tau(m)), m$ be the dimensionless molecular weight $m=M / M_{0}$, where $M_{0}$ is the monomer molecular weight and $M$ is the molecular weight of the polymer. The above relationships are valid in the molecular weight range: $m_{e}<m<\infty$, where $m_{e}$ denotes the entanglement molecular weight.

The generalized mixing rule, used in the derivation of the relationship Eq.(11), was taken to have the form

$$
G(t)=G_{N}^{0}\left(\int_{m_{e}}^{\infty} F^{1 / \beta}(t, m) \frac{w(m)}{m} \mathrm{~d} m\right)^{\beta} .
$$

The value for $\beta$ can be determined theoretically from polymer dynamical considerations. It takes the value 1 for single reptation (Doi and Edwards (1986)), and 2 for double reptation or entanglement (Tsenoglou (1987,1991), Des Cloizeaux (1988)). Thimm et al. (2000) have shown that a value of $\beta \approx 2$ can be justified, if the Rouse spectrum is treated correctly in the evaluation of rheological 
data. The relaxation processes of the individual polymer chains (reptation, double reptation) with molecular weight $m$, which correspond to the experimentally determined relaxation shear modulus $G(t)$, are described by the integral kernel $F(t, m)$. Based on either polymer dynamical considerations or phenomenological observations, five kernels have been examined and discussed in the literature (see e.g. Wasserman and Graessly (1992), Maier et al. (1998)): single exponential; Tuminello; Doi; Des Cloizeaux and BSW (Baumgärtel-Schausberger-Winter). All of these kernels do not depend on the nature of the polymer being investigated. In Maier et al. (1998) the single exponential kernel gave the best agreement with experimental observations.

Keeping the generality of the mixing rule Eq. (州 it is possible to derive a kernel, which depends on $w(m)$ and has the single exponential kernel as the limit for monodisperse distributions. The derivation of this kernel is discussed in section II. The physical relevance of the kernel is discussed in section III.

\section{Derivation of the MWD-dependent kernel}

The decomposition of rheological material functions, such as the relaxation shear modulus $G(t)$, in terms of Maxwell-modes is an accepted procedure to present results of rheological measurements (see e.g. Ferry (1980)). The following equation combines the relaxation shear modulus $G(t)$ with the corresponding relaxation time spectrum $h(\tau)$ :

$$
G(t)=\int_{0}^{\infty} \frac{h(\tau)}{\tau} e^{-t / \tau} \mathrm{d} \tau
$$

The equilibrium shear modulus $G_{e}$ is assumed to be zero for the viscoelastic liquids under consideration. Whereas a decomposition in terms of other kernels is possible too, the use of Eq. (5) has been established empirically.

We summarize the derivation of the results found in Thimm et al. (1999), where the definition Eq. (5) of the relaxation time spectrum was combined with 
the mixing rule Eq. (4) above. The algebraic transformation:

$$
\int_{a}^{\infty} \mathrm{d} x\left(-\frac{\mathrm{d}}{\mathrm{d} x}\right)\left[\int_{x}^{\infty} f\left(x^{\prime}\right) d x^{\prime}\right]^{\gamma}=\left[\int_{a}^{\infty} f(x) d x\right]^{\gamma}=\gamma \int_{a}^{\infty} f(x)\left[\int_{x}^{\infty} f\left(x^{\prime}\right) d x^{\prime}\right]^{\gamma-1} d x
$$

was applied on Eq. (5):

$$
\begin{aligned}
G(t)^{1 / \beta}= & \left(\int_{\tau_{e}}^{\infty} \frac{h(\tau)}{\tau} e^{-t / \tau} d \tau\right)^{1 / \beta}=\left(\int_{m_{e}}^{\infty} \alpha \frac{\tilde{h}\left(m^{\prime}\right)}{m^{\prime}} e^{-t / \tau\left(m^{\prime}\right)} \mathrm{d} m^{\prime}\right)^{1 / \beta}= \\
& \int_{m_{e}}^{\infty} \frac{\alpha^{(1 / \beta)}}{\beta} \frac{\tilde{h}(m)}{m} e^{-t / \tau(m)}\left[\int_{m}^{\infty} \frac{\tilde{h}\left(m^{\prime}\right)}{m^{\prime}} e^{-t / \tau\left(m^{\prime}\right)} \mathrm{d} m^{\prime}\right]^{(1 / \beta)-1} \mathrm{~d} m .
\end{aligned}
$$

and the result inserted in Eq. (4). The following relation connecting the relaxation time spectrum and the MWD $w(m)$ was found:

$$
\begin{array}{r}
\int_{m_{e}}^{\infty}\left[\frac{a^{(1 / \beta)}}{\beta} \frac{\tilde{h}(m)}{m} e^{-t / \tau(m)}\left[\int_{m}^{\infty} \frac{\tilde{h}\left(m^{\prime}\right)}{m^{\prime}} e^{-t / \tau\left(m^{\prime}\right)} \mathrm{d} m^{\prime}\right]^{(1 / \beta)-1}\right. \\
\left.-\left(G_{N}^{0}\right)^{1 / \beta} F(t, m)^{1 / \beta} \frac{w(m)}{m}\right] \mathrm{d} m=0 .
\end{array}
$$

One solution, which fulfills Eq. (8) is that the kernel under the integral is identical to zero:

$$
\begin{array}{r}
\frac{a^{(1 / \beta)}}{\beta} \tilde{h}(m) e^{-t / \tau(m)}\left[\int_{m}^{\infty} \frac{\tilde{h}\left(m^{\prime}\right)}{m^{\prime}} e^{-t / \tau\left(m^{\prime}\right)} \mathrm{d} m^{\prime}\right]^{(1 / \beta)-1} \\
-\left(G_{N}^{0}\right)^{1 / \beta} F(t, m)^{1 / \beta} w(m)=0 .
\end{array}
$$

For the time $t=0$, we have $F(t=0, m)=1$, because $G(t=0)=G_{N}^{0}$ and the integral over the molecular weight distribution in the mixing rule Eq. 1 becomes one.

Hence we derive from Eq. (9) for $t=0$ the equation (11). When the analytical relation (11) is inserted in equation (9), the constants (i.e. $\alpha, G_{N}^{0}$ ) cancel and one finds that

$$
\exp (-t / \tau)\left[\int_{m}^{\infty} \frac{\tilde{h}\left(m^{\prime}\right)}{m^{\prime}} \exp \left(-t / \tau\left(m^{\prime}\right) \mathrm{d} m^{\prime}\right]^{1 / \beta-1}=F(t, m)^{1 / \beta}\left[\int_{m}^{\infty} \frac{\tilde{h}\left(m^{\prime}\right)}{m^{\prime}} \mathrm{d} m^{\prime}\right]^{1 / \beta-1} .\right.
$$


This equation can be reordered (using Eq. (2)) to find an integral kernel, which depends implicitly on the molecular weight distribution:

$$
F(t, m)=\exp (-t / \tau(m))^{\beta}\left[\frac{\int_{m}^{\infty} \frac{w\left(m^{\prime \prime}\right)}{m^{\prime \prime}}\left(\int_{m^{\prime \prime}}^{\infty} \frac{w\left(m^{\prime}\right)}{m^{\prime}} \mathrm{d} m^{\prime}\right)^{\beta-1} \exp \left(-t / \tau\left(m^{\prime \prime}\right)\right) \mathrm{d} m^{\prime \prime}}{\int_{m}^{\infty} \frac{w\left(m^{\prime \prime}\right)}{m^{\prime \prime}}\left(\int_{m^{\prime \prime}}^{\infty} \frac{w\left(m^{\prime}\right)}{m^{\prime}} \mathrm{d} m^{\prime}\right)^{\beta-1} \mathrm{~d} m^{\prime \prime}}\right]^{1-\beta} .
$$

When $\beta=2$ (double reptation) is inserted in Eq. (11), one finds that

$$
F(t, m)=\exp (-t / \tau(m)) \frac{\exp (-t / \tau(m)) \int_{m}^{\infty} \frac{w\left(m^{\prime \prime}\right)}{m^{\prime \prime}}\left(\int_{m^{\prime \prime}}^{\infty} \frac{w\left(m^{\prime}\right)}{m^{\prime}} \mathrm{d} m^{\prime}\right) \mathrm{d} m^{\prime \prime}}{\int_{m}^{\infty} \frac{w\left(m^{\prime \prime}\right)}{m^{\prime \prime}}\left(\int_{m^{\prime \prime}}^{\infty} \frac{w\left(m^{\prime}\right)}{m^{\prime}} \mathrm{d} m^{\prime}\right) \exp \left(-t / \tau\left(m^{\prime \prime}\right)\right) \mathrm{d} m^{\prime \prime}} .
$$

For monodisperse distributions the result that the kernel is of single exponential type (single exponential kernel) is easily reconstructed (inserting Dirac's $\delta$ function: $w(m) / m=\delta\left(m-m_{0}\right)$, where the integration over the $\delta$-function gives 1). This observation agrees with the result, that the single exponential kernel describes the data best, found by Maier et al. (1998) evaluating rheological data of polystyrene mixtures. For other MWDs Eq.'s (11, 12) establish a new kind of integral kernel, to be used in the mixing rule Eq. (代).

In the next section some features of the proposed kernel are discussed.

\section{Discussion}

The physical interpretation of the kernel is that the relaxation of a single polymer seems to be implicitly dependent on the molecular weight distribution of the neighbouring polymers. While for a monodisperse MWD the result of the single exponential kernel is found, the kernel predicts a more complex behaviour for highly polydisperse polymer melts.

There are several contributions in literature (e.g. Graessly (1982), Montfort et al. (1984), Rubinstein and Colby (1987), McLeish(1992)), which discuss that the relaxation behaviour is different in monodisperse and polydisperse samples. The relaxation behaviour is accelerated in polydisperse samples. The reason is that when a short chain reptates away, the entanglements between long chains become 
ineffective. The short chains are considered as solvent, which dilute the density of entanglements in the polymer melt and therefore lead to an effective faster relaxation process. The proposed kernel agrees qualitatively with this picture.

The relaxation behaviour of the kernels is illustrated in Fig. 1. We have used a monomodale log-normal distribution with common parameters and $\beta=2$ to simulate the data in the shown figures. We find that the relaxation is faster in the highly polydisperse than in the near monodisperse sample.

When the kernel is inserted in the mixing rule Eq.(四), it is found that the main influence of the molecular weight distribution on $G(t)$ is due to the integration over $w(m)$, whereas the MWD in the kernels has a smaller influence on $G(t)$. In Fig. 2 are several $G(t)$ plotted, which were simulated with either the pure exponential or with the proposed kernel. The $G(t)$ curves are shifted mainly parallel to each other, but there are no additional structural changes besides the shift. One finds that the main influence of the MWD on $G(t)$ is independent of the kernel's details. This observation is in agreement with the idea introduced by Thimm et al. (1999), that the molecular weight distribution can be determined from rheological data without explicitly regarding the details of the kernels.

To achieve a better understanding of Eq. (12), we approximate the exponential in the integral by a step function $(\exp (-t / \tau(m)=\theta(\tau(m)-t))$, where $\theta(\tau(m)-t)=1$ for $t<\tau(m)$ and $\theta(\tau(m)-t)=0$ for $t>\tau(m))$. This approximation leads to a lower limit of the integral in Eq. (12), which depends on the time and is denoted by $\tilde{m}(t)$. With this simplification, one can use Eq. (6) and obtains a simpler form of the kernel:

$$
F(t, m)=\left[\exp (-t / \tau) \frac{\int_{m}^{\infty} \frac{w\left(m^{\prime}\right)}{m^{\prime}} \mathrm{d} m^{\prime}}{\int_{\tilde{m}(t)}^{\infty} \frac{w\left(m^{\prime}\right)}{m^{\prime}} \mathrm{d} m^{\prime}}\right]^{2} .
$$

Inserting this kernel in the mixing rule (4), one finds (for $\beta=2$ ) that:

$$
G(t)=G_{N}^{0}\left[\int_{m_{e}}^{\infty} \exp (-t / \tau(m))\left[\frac{\int_{m}^{\infty} \frac{w\left(m^{\prime}\right)}{m^{\prime}} \mathrm{d} m^{\prime}}{\int_{\tilde{m}(t)}^{\infty} \frac{w\left(m^{\prime}\right)}{m^{\prime}} \mathrm{d} m^{\prime}}\right] \frac{w(m)}{m} \mathrm{~d} m\right]^{2} .
$$


Besides the single exponential behaviour another term is found (in brackets), which depends implicitly on the MWD and the time.

The mathematical details of the integration over MWD as in Eq. (13) using typical MWDs as ansatz have been discussed by Eder et al. (1989). We do not think such details relevant for the study discussed in this article, but emphazise that the analytical relations (1, 2) are valid independent of any assumptions concerning the MWD.

\section{Conclusion}

We have proposed a new kernel, which is implicitly dependent on the molecular weight distribution. In the limit of a monodisperse sample this kernel contains the well-known result of the single exponential kernel as special case. The physical interpretation of this kernel is that the relaxation of a single polymer seems to be implicitly dependent on the molecular weight distribution of the neighbouring polymers.

This observation enlarges the physical features of the class of mixing rules discussed by Anderssen and Mead (1998) in a new way. The advantage of this implicitly molecular weight distribution dependent kernel is that the physics (the reptation processes) described by the kernels can be fine tuned but the general form of the mixing rule discussed by Anderssen and Mead (1998) stays the same.

\section{Acknowledgement}

We thank M. Marth for helpful discussions and R. S. Anderssen for assistance in formulation of the manuscript. W. B. Thimm was supported by the Deutsche Forschungsgemeinschaft: Graduiertenkolleg für Strukturbildung in makromolekularen Systemen. 


\section{References}

Anderssen R. S. and D. W. Mead "Theoretical derivation of molecular weight scaling for rheological parameters," J. Non-Newtonian Fluid Mech. 76, 299-306 (1998).

Des Cloizeaux, J., "Double reptation vs simple reptation in polymer melts," Europhys. Lett. 5, 437-442 (1988); 6, 475 (1988).

Doi, M. and S. F. Edwards, The Theory of Polymer Dynamics, (Clarendon, Oxford 1986).

Eder G., Janeshitz-Kriegl H., Liedauer S., Schausberger A., Stadlbauer W., "The influence of Molar Mass Distribution on the Complex Moduli of Polymer Melts", J. Rheol. 33(6), 805-820 (1989)

Ferry, F. D., Viscoelastic Properties of Polymers, 3rd ed. (Wiley, New York 1980).

Graessly, W. W., "Entangled linear, branched and Network Polymer systems molecular theories," Adv. Polym. Sci. 47 , 67-117 (1982)

Maier, D., A. Eckstein, C. Friedrich, J. Honerkamp, "Evaluation of Models Combining Rheological Data with the Molecular Weight distribution," J. Rheol. 42(5), 1153-1173 (1998)

Montfort, J.-P., Marin, G., Monge, P., "Effects of constraint release on the dynamics of entangled linear polymer melts," Macromolecules 17, 1551-1560 (1984)

McLeish, T. C. B., "Relaxation behaviour of highly polydisperse polymer melts," 
Polymer 33 (13), 2852-2854 (1992)

Rubinstein, M., Colby R. H., "Self-consistent theory of polydisperse entangled polymers: Linear viscoelasticity of binary blends," J. Chem. Phys. 89 (8), 5291$5306(1988)$

Thimm, W., C. Friedrich, M. Marth, J. Honerkamp, "An Analytical Relation between Relaxation Time Spectrum and Molecular Weight Distribution," J. Rheol. 43(6), 1663-1672 (1999)

Thimm, W., C. Friedrich, M. Marth, J. Honerkamp, "On the Rouse spectrum and the determination of the molecular weight distribution," J. Rheol. 44(2), $429-438(2000)$

Tsenoglou, C., "Viscoelasticity of binary homopolymer blends," ACS Polym. Prepr. 28, 185-186 (1987).

Tsenoglou, C., "Molecular Weight Polydispersity Effects on the Viscoelasticity of Entangled Linear Polymers," Macromolecules 24, 1762-1767 (1991).

Wasserman, S. H. and W. W. Graessley, "Effects of polydispersity on linear viscoelasticity in entangled polymer melts," J. Rheol. 36, 543-572 (1992). 


\section{FIG. 1}

For various polydisperse molecular weight distributions $(\mathrm{Mw}=300 \mathrm{~kg} / \mathrm{mol}, \mathrm{Ip}=$ $\mathrm{Mw} / \mathrm{Mn}=1.2,8)$ the behaviour of the proposed kernel at fixed $M \approx 1000 \mathrm{~kg} / \mathrm{mol}$ is illustrated. Solid lines: single exponential kernel, dashed line $(\mathrm{Ip}=1.2)$, dotted line $(\mathrm{Ip}=8)$.

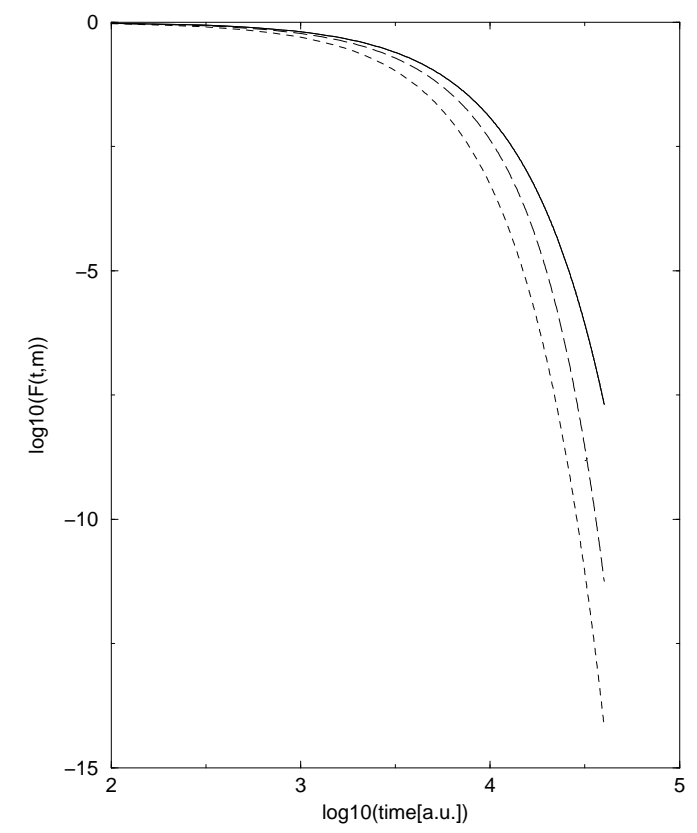




\section{FIG. 2}

For two polydisperse molecular weight distributions $\mathrm{Ip}=1.2$ and $8, \mathrm{Mw}=300$ $\mathrm{kg} / \mathrm{mol}$, the behaviour of $G(t) / G_{N}^{0}$ is illustrated. The two different kernels are distinguished: single exponential kernel (solid), the implicitly molecular weight distribution dependent kernel (dashed).

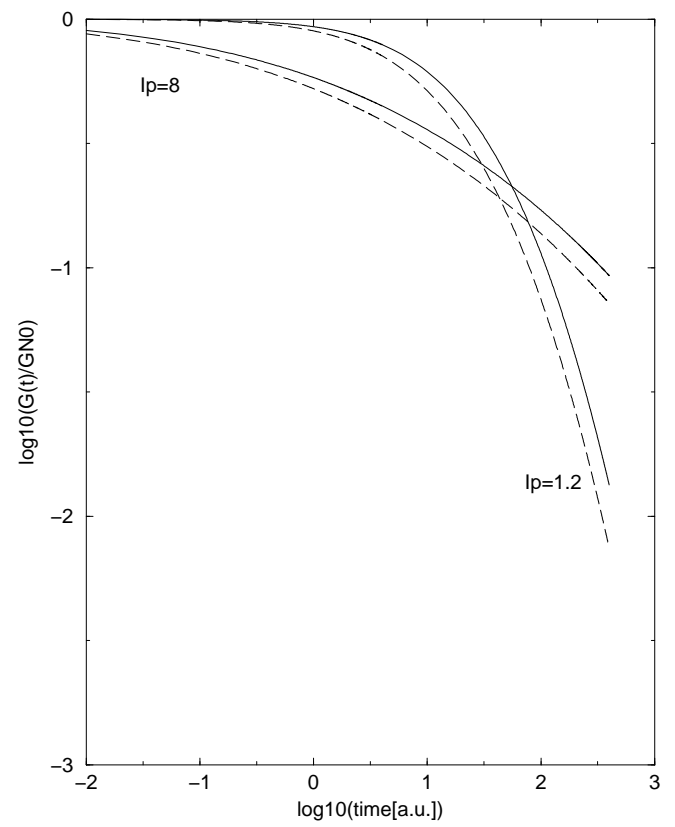

